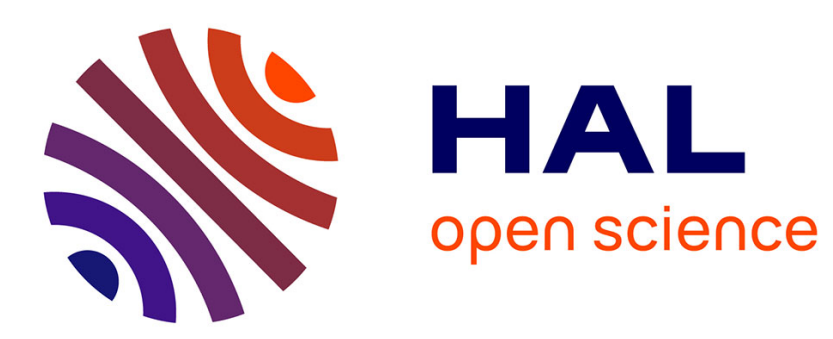

\title{
Multimodal Standing Gravity Waves: a Completely Resonant System
}

\author{
Gérard Iooss, Pavel Plotnikov
}

\section{To cite this version:}

Gérard Iooss, Pavel Plotnikov. Multimodal Standing Gravity Waves: a Completely Resonant System. Journal of Mathematical Fluid Mechanics, 2005, 7 (S), pp.17. 10.1007/s00021-004-0128-4 . hal01265187

\section{HAL Id: hal-01265187 \\ https://hal.univ-cotedazur.fr/hal-01265187}

Submitted on 31 Jan 2016

HAL is a multi-disciplinary open access archive for the deposit and dissemination of scientific research documents, whether they are published or not. The documents may come from teaching and research institutions in France or abroad, or from public or private research centers.
L'archive ouverte pluridisciplinaire HAL, est destinée au dépôt et à la diffusion de documents scientifiques de niveau recherche, publiés ou non, émanant des établissements d'enseignement et de recherche français ou étrangers, des laboratoires publics ou privés. 


\title{
Multimodal standing gravity waves: a completely resonant system.
}

\author{
Gérard Iooss $^{\dagger}$, Pavel Plotnikov \\ † IUF, INLN UMR 6618 CNRS - UNSA, 1361 rte des Lucioles, 06560 Valbonne, France \\ gerard.iooss@inln.cnrs.fr \\ $\ddagger$ Russian academy of Sciences, Lavryentyev pr. 15, Novosibirsk 630090, Russia.
}

plotnikov@hydro.nsc.ru

\begin{abstract}
The standing gravity wave problem on an infinitely deep fluid layer is considered under the form of a nonlinear non local scalar PDE of second order as in [6]. Nonresonance at quadratic order of the infinite dimensional bifurcation equation, allows to give the explicit form of the quadratic change of variables able to suppress quadratic terms in the nonlinear equation. We state precisely the equivalence between formulations in showing that the above unbounded change of variable is invertible.

The infinite set of solutions which can be expanded in powers of amplitude $\varepsilon$ is then given up to order $\varepsilon^{2}$.

Key words: nonlinear water waves, standing gravity waves, bifurcation theory, complete resonance.

AMS classification: 35B32, 35B34, 76B15, 76B07
\end{abstract}

\section{Introduction}

This is the first of a series of two papers concerning the existence of two-dimensional standing gravity water waves on an infinitely deep perfect fluid layer, non necessarily "unimodal" as in [6]. The nonlinear problem of existence of small amplitude standing gravity waves ("clapotis" in french) comes back to Boussinesq (1877), Rayleigh (1915), Sekerkh-Zenkovich (1947), Schwartz and Whitney [8] (1981), Amick and Toland [1] (1987) (see historical references in [6]) who gave the asymptotic expansion of unimodal standing waves (only one dominant mode at the main order $\varepsilon$ ) respectively at order $\varepsilon^{2}, \varepsilon^{3}, \varepsilon^{4}$, conjectured an algorithm for computing all orders [8], and finally showed that this algorithm indeed works [1].

For multimodal standing waves, which means that at order $\varepsilon$ there is a suitable combination of several modes, their existence is presumed, with no justification by Penney and Price in [7] (see p.260), while much later a footnote p.168 in [8] claims after a significant discussion during the review process, that they are not expected to exist, based on the possible occurence of secular terms at order $\varepsilon^{3}$. However, 13 years later, there were numerical evidence (see Bryant and Stiassnie [2]) of the possibility to build the beginning (up to order $\left.\varepsilon^{100}\right)$, of asymptotic expansions. Finally, the possibility to find a large family of approximate solutions for our problem, in the form of asymptotic expansions in powers of the amplitude $\varepsilon$, was proved in [5]. The point here is to prove that all these asymptotic expansions indeed correspond to standing waves, each one existing for a set of values of $\varepsilon$ having 0 as a Lebesgue point, i.e. dense at 0 . Since we need to use a scalar formulation different from the one used in [5], we need to give precisely on this formulation, the formal asymptotic expansions which serve as a basis for the proof of the existence of solutions. The general form of these asymptotic expansions is deduced from a new formulation of the problem 
obtained after a quadratic "normalizing" change of variable which is precisely studied here, showing an equivalence between formulations. The second paper strictly follows the proof made in [6] for the unimodal case, for showing the existence of all solutions which possess these asymptotic expansions.

After scaling, there is one dimensionless parameter $1+\mu=g T^{2} / 2 \pi \lambda$ where $g$ is the acceleration of gravity, $T$ is the time period, $\lambda$ is the horizontal wave length. Due to a scaling invariance in the problem, we may, without loss of generality, assume that $\mu$ is close to 0 . We indeed look for non trivial doubly $2 \pi$ - periodic solutions of the following second order nonlocal PDE, as deduced from the formulation introduced by Dyachenko et al [4]:

$$
\partial_{t}\left(L_{w^{\prime}} \dot{w}\right)-(1+\mu) \mathcal{H} w^{\prime}+\mathcal{H} \partial_{x}\left\{\frac{1}{D} \mathcal{H}\left(\left(L_{w^{\prime}} \dot{w}\right) \mathcal{H} L_{w^{\prime}} \dot{w}\right)+\left(\mathcal{H} L_{w^{\prime}} \dot{w}\right) \mathcal{H}\left(\frac{L_{w^{\prime}} \dot{w}}{D}\right)\right\}=0
$$

where $w$ is an unknown function of $(x, t) \in \mathbb{R}^{2}$, the free surface of the waves being given in the physical plane by

$$
(\xi, \eta)=(x+\mathcal{H} w(x, t),-w(x, t)), \quad(x, t) \in \mathbb{R}^{2} .
$$

In (1) the linear operator $\mathcal{H}$ denotes the periodic Hilbert transform with respect to $x$

$$
\mathcal{H}(\cos n x)=-\sin |n| x, \mathcal{H}(\sin n x)=\operatorname{sgn}(n) \cos n x \quad(n \neq 0),
$$

we denote by a dot or $\partial_{t}$ the time $t$ partial derivative and by a prime or $\partial_{x}$ the space $x$ partial derivative. The function $D$ and the linear operator $L_{w^{\prime}}$ are defined as follows, for $w$ doubly periodic and smooth enough and any $f \in L_{\natural}^{2}=L^{2}(\mathbb{R} / 2 \pi \mathbb{Z})$

$$
\begin{aligned}
L_{w^{\prime}} f & =\left(1+\mathcal{H} w^{\prime}\right) f-w^{\prime} \mathcal{H} f \\
D & =\left(1+\mathcal{H} w^{\prime}\right)^{2}+w^{\prime 2} .
\end{aligned}
$$

We look for solutions of (1) which are even in $x$ and in $t$ (this is for eliminating solutions deduced by shifting time origin and by translation in $x$ ). Moreover, since adding a constant to $w$ gives a new solution of the problem, we restrict our study to solutions $w$ with 0 average (they correspond to the same physical solutions). Equation (1) may be written as

$$
\mathcal{F}(w, \mu)=0,
$$

where $\mathcal{F}$ is a analytic mapping $H_{\text {如 }}^{m, e e} \times \mathbb{R} \rightarrow H_{\text {响 }}^{m-2, e e}, m \geq 3$, where $H_{\text {响 }}^{m, e e}$ is by definition the subspace of functions even in $x$ and in $t$ of the Sobolev space $H_{\text {响 }}^{m}=H^{m}\left\{(\mathbb{R} / 2 \pi \mathbb{Z})^{2}\right\}$. Defining the nonlinear terms $\mathcal{N}_{\geq 2}$, the quadratic terms $\mathcal{N}_{2}$ and the terms of at least cubic order $\mathcal{N}_{\geq 3}$ we can write

$$
\begin{aligned}
\mathcal{F}(w, \mu) & =\mathcal{L}_{0} w-\mu \mathcal{H} w^{\prime}+\mathcal{N}_{\geq 2}(w) \\
& =\mathcal{L}_{0} w-\mu \mathcal{H} w^{\prime}+\mathcal{N}_{2}(w, w)+\mathcal{N}_{\geq 3}(w)
\end{aligned}
$$

where

$$
\mathcal{L}_{0} w=\ddot{w}-\mathcal{H} w^{\prime} .
$$

The kernel of $\mathcal{L}_{0}$ is infinite dimensional, spanned by

$$
\left\{\cos q^{2} x \cos q t ; \quad q \in \mathbb{N}\right\}
$$

One says that the problem is completely resonant, and this leads to an infinite dimensional bifurcation equation. We show below that there are infinitely many formally bifurcating solutions near $\mu=0$ (see theorem 5 ), where formally means that we can compute infinitely many different expansions in powers of the amplitude $\varepsilon$, where $\mu=\varepsilon^{2} / 4$, which are formal 
solutions of (1). To prove such a result, we use a new formulation of the problem where all quadratic terms in (2) no longer exist. This formulation results from a simple (unbounded) quadratic change of variables (see Lemma 1). We then give the invertibility properties of this mapping, which insures the equivalence between both formulations (see Theorem 2).

Acknowledgements The authors deeply acknowledge John Toland for many fruitful discussions.

\section{Bifurcation equation}

Because of the basic non resonance property

$$
\left.\int_{-\pi}^{\pi} \int_{-\pi}^{\pi}\left\{\exp i\left[q^{2} \pm p^{2} \pm r^{2}\right) x+( \pm q \pm p \pm r) t\right]\right\} d x d t=0
$$

which is valid for any positive integers $p, q, r$, the quadratic term of the bifurcation equation cancels:

$$
P_{0} \mathcal{N}_{2}(\Theta, \Theta) \equiv 0
$$

for any $\Theta \in \operatorname{ker} \mathcal{L}_{0}$, and where $P_{0}$ is the orthogonal projection in $L_{\text {如 }}^{2}$ on ker $\mathcal{L}_{0}$. Instead of using directly the Lyapunov-Schmidt method on the formulation $(1)$, let us use the possibility of finding a quadratic change of variables which kills the quadratic terms in (1). The possibility of finding such a quadratic change of variables is a consequence of property (5), and was explicitely given in [5] with another formulation of the problem which exploits the "holomorphic" part of the formulation, and originally exploits a Schwartz-Whitney conjecture (see [1], [5]). In principle such a change of variable can be computed in using Fourier series of quadratic terms in (1), and looking for a quadratic unknown change of variable under the form of its Fourier series. However, because of the not very complicated form of the quadratic terms in (1), we can also try to "guess" the change of variable, since it is simple, in using combinations of all possible monomials containing the operator $\mathcal{H}$, such that the evenness property of $w$ is conserved.

Once this change is done, it is clear that the principal part of the (infinite dimensional) bifurcation equation (linear and cubic terms), will be simpler to compute. Indeed, we can show the following (below a term like $O\left(\|v\|^{p}\right)$ means formally that multiplying $v$ by $\lambda$ gives a term of order $O\left(\lambda^{p}\right)$ when $\lambda \rightarrow 0$ )

Lemma 1 The analytic mapping $F: H_{\text {柷 }}^{m} \rightarrow H_{\text {吼 }}^{m-1}, m>2$, defined by

$$
v=w+B\left(w, w^{\prime}\right) \stackrel{\text { def }}{=} F(w)
$$

with

$$
B\left(w_{1}, w_{2}\right)=\mathcal{H}\left(w_{1} w_{2}\right)-w_{1} \mathcal{H} w_{2}-2 w_{2} \mathcal{H} w_{1}
$$

defines, for sufficiently smooth doubly periodic $w$, a formal change of variables which changes equation (2) into a new equation $\mathcal{E}(v, \mu)=0$ with no quadratic term in $v$. More precisely we obtain

$$
\begin{aligned}
\mathcal{E}(v, \mu) & =\mathcal{F}(w, \mu)+\partial_{x} B(w, \mathcal{F}(w, \mu)) \\
\mathcal{F}(w, \mu) & =\widetilde{\Gamma}_{w} \mathcal{E}(v, \mu)
\end{aligned}
$$

where

$$
\begin{aligned}
& \mathcal{E}(v, \mu) \stackrel{\text { def }}{=} \mathcal{L}_{0} v-\mu \mathcal{H} v^{\prime}+\mathcal{N}_{\geq 3}(G(v))+\partial_{x} B\left(G(v), \mathcal{N}_{\geq 2}(G(v))\right) \\
= & \mathcal{L}_{0} v-\mu \mathcal{H} v^{\prime}+\mathcal{N}_{3}(v, v, v)+\partial_{x} B\left(v, \mathcal{N}_{2}(v, v)\right)+O\left(\|v\|^{4}\right)
\end{aligned}
$$


$G$ is the inverse of $F$ (see the theorem below for the properties of $G$ )

$$
G(v)=v-B\left(v, v^{\prime}\right)+B\left(B\left(v, v^{\prime}\right), v^{\prime}\right)+B\left(v, \partial_{x} B\left(v, v^{\prime}\right)\right)+O\left(\|v\|^{4}\right),
$$

and $\widetilde{\Gamma}_{u}$ is the linear operator defined formally by (see the properties of $\widetilde{\Gamma}_{u}$ below)

$$
\begin{aligned}
e & =f+\partial_{x} B(u, f) \Leftrightarrow f=\widetilde{\Gamma}_{u} e \\
\widetilde{\Gamma}_{u} & =\mathbb{I}-\partial_{x} B(u, \cdot)+\partial_{x} B\left(u, \partial_{x} B(u, \cdot)\right)+O\left(\|u\|^{3}\right), \\
\partial_{u} \widetilde{\Gamma}_{u}[\delta u] & =-\widetilde{\Gamma}_{u} \partial_{x} B\left(\delta u, \widetilde{\Gamma}_{u} \cdot\right) .
\end{aligned}
$$

Remark: Notice that $F: H_{\text {吅 }}^{s} \rightarrow H_{\text {吅 }}^{s-1}$ is an analytic mapping for any $s>2$. We also notice that adding a constant to $w$ corresponds to adding the same constant to $v$. Hence for the solution $v$ we can restrict to the $v$ with zero average.

Moreover, we have the following smoothness property for the change of variable:

Theorem 2 Fix an arbitrary $p>3$ and integer $n \geq 1$. Then there is $r(p)>0$, and $a$ mapping

$$
G: B_{p}(r)=\left\{v \in H_{\text {如 }}^{p} ;\|v\|_{H^{p}} \leq r(p)\right\} \rightarrow H_{\text {如 }}^{p}
$$

such that

(a) $F(G(v))=v$ for each $v \in B_{p+n}(r)$;

(b) $\|G(v)\|_{H^{p}} \leq 2\|v\|_{H^{p}}$;

whenever $v, v+h \in B_{p+n}(r), r<r(p+n)$,

$$
\begin{aligned}
G(v+h)-G(v) & =\sum_{k=1}^{n} G_{k}(v)(h)+O_{n}(v, h), \\
\left\|O_{n}(v, h)\right\|_{H^{p-1}} & =O\left(\|h\|_{H^{p+n-1}}^{n+1}\right) \text { as } h \rightarrow 0 .
\end{aligned}
$$

The $k$-linear operators $G_{k}(v): H_{\text {如 }}^{s+k-1} \rightarrow H_{\text {如 }}^{s}$, for any $s$ such that $2<s \leq p+n-k$ have the bounds

$$
\left\|G_{k}(v)(h)\right\|_{H^{s}} \leq c(n, p)\|h\|_{H^{s+k-1}}^{k}
$$

and are defined by the equalities

$$
G_{k}(v)(h)=\partial_{w} F(w)^{-1} \mathfrak{R}_{k}(h), \quad w=G(v),
$$

in which

$$
\begin{gathered}
\partial_{w} F(w) q \stackrel{\text { def }}{=} q+\mathcal{H}\left(w q^{\prime}\right)-w \mathcal{H} q^{\prime}+\mathcal{H}\left(q w^{\prime}\right)-q \mathcal{H} w^{\prime}-2 q^{\prime} \mathcal{H} w-2 w^{\prime} \mathcal{H} q, \\
\mathfrak{R}_{1}(h)=h, \quad \mathfrak{R}_{k}(h)=-\sum_{\substack{i+j=k, 1 \leq i, j \leq n}} B\left(G_{i}(h), G_{j}(h)^{\prime}\right) \text { for } k>1
\end{gathered}
$$

In particular, $G_{1}:=\partial_{v} G(v)=\partial_{w} F(w)^{-1}$.

Corollary 3 For $w \in H_{\text {耴 }}^{s+1}$ such that $\|w\|_{H^{s+1}} \leq r(s)$, the linear operator $\widetilde{\Gamma}_{w}$ is bounded in $H_{\text {如 }}^{s}$ for $s>2$ and satisfies

$$
\left\|\widetilde{\Gamma}_{w}\right\|_{\mathcal{L}\left(H^{s}\right)} \leq c, \quad\left\|\widetilde{\Gamma}_{w} e-e\right\|_{H^{s-1}} \leq c\|w\|_{H^{s+1}}\|e\|_{H^{s}}
$$

The proofs of theorem 2 and corollary 3 are in Appendix 2 .

It then follows immediately the following smoothness properties for $\mathcal{E}(v, \mu)$ : 
Corollary 4 For $p>3$ there exists $r(p)>0$ such that for $\|v\|_{H^{p}} \leq r(p)$, the map $(v, \mu) \mapsto$ $\mathcal{E}(v, \mu)$ is defined from $H_{\text {响 }}^{p} \times \mathbb{R}$ to $H_{\text {吅 }}^{p-3}$, being analytic in $\mu$. Moreover, for positive integer $k$ and $p>3+k$, and $v \in H_{\text {吆 }}^{p}$, the (Gâteaux-) derivative $\partial_{v}^{k} \mathcal{E}(v, \mu)$ is a bounded $k$-linear operator from $H_{\text {如 }}^{p}$ to $H_{\text {如 }}^{p-k-2}$.

Remark: The above theorem and corollaries show that in a neighborhood of 0 , the solutions $v$ of $\mathcal{E}(v, \mu)=0$ correspond exactly through the mapping $F$ to the solutions of $\mathcal{F}(w, \mu)=0$, provided that these solutions are smooth enough.

Proof of the lemma: this is based on the following properties of $B$ :

Property 1: For smooth enough $w_{1}$ and $w_{2}$ such that $\pi_{0} w_{2}=\pi_{0}\left(w_{1} w_{2}\right)=0$, then

$$
\mathcal{H} B\left(w_{1}, w_{2}\right)=B\left(w_{1}, \mathcal{H} w_{2}\right) .
$$

Property 2:

$$
B\left(\ddot{w}, w^{\prime}\right)+2 B\left(\dot{w}, \dot{w}^{\prime}\right)-B\left(w^{\prime}, \ddot{w}\right)=\mathcal{N}_{2}(w, w) .
$$

Assume for the moment, that these two properties hold. Then we can compute (since $\left.\pi_{0} w^{\prime}=\pi_{0}\left(w w^{\prime}\right)=0\right)$

$$
\begin{aligned}
\mathcal{L}_{0} v-\mu \mathcal{H} v^{\prime} & =\mathcal{L}_{0} w-\mu \mathcal{H} w^{\prime}+B\left(\ddot{w}, w^{\prime}\right)+2 B\left(\dot{w}, \dot{w}^{\prime}\right)+B\left(w, \ddot{w}^{\prime}\right)-(1+\mu) \partial_{x} B\left(w, \mathcal{H} w^{\prime}\right) \\
& =\mathcal{L}_{0} w-\mu \mathcal{H} w^{\prime}+\mathcal{N}_{2}(w, w)+\partial_{x} B\left(w, \mathcal{L}_{0} w-\mu \mathcal{H} w^{\prime}\right) \\
& =\mathcal{F}(w, \mu)-\mathcal{N}_{\geq 3}(w)+\partial_{x} B\left(w, \mathcal{F}(w, \mu)-\mathcal{N}_{\geq 2}(w)\right)
\end{aligned}
$$

hence

$$
\mathcal{L}_{0} v-\mu \mathcal{H} v^{\prime}+\mathcal{N}_{\geq 3}(w)+\partial_{x} B\left(w, \mathcal{N}_{\geq 2}(w)\right)=\mathcal{F}(w, \mu)+\partial_{x} B(w, \mathcal{F}(w, \mu))
$$

which is the result of the lemma, once we notice that formally

$$
G(v)=v+O\left(\|v\|^{2}\right) .
$$

It remains to prove properties 1 and 2 .

Proof of property 1: we have

$$
\begin{aligned}
\mathcal{H} B\left(w_{1}, w_{2}\right) & =-w_{1} w_{2}+\pi_{0}\left(w_{1} w_{2}\right)-\mathcal{H}\left(w_{1} \mathcal{H} w_{2}\right)-2 \mathcal{H}\left(w_{2} \mathcal{H} w_{1}\right) \\
B\left(w_{1}, \mathcal{H} w_{2}\right) & =\mathcal{H}\left(w_{1} \mathcal{H} w_{2}\right)+w_{1}\left(w_{2}-\pi_{0} w_{2}\right)-2\left(\mathcal{H} w_{2}\right)\left(\mathcal{H} w_{1}\right)
\end{aligned}
$$

and the identity

$$
\mathcal{H}\left(w_{1} \mathcal{H} w_{2}\right)+\mathcal{H}\left(w_{2} \mathcal{H} w_{1}\right)-\left(\mathcal{H} w_{1}\right)\left(\mathcal{H} w_{2}\right)+w_{1} w_{2}=\left(\pi_{0} w_{1}\right)\left(\pi_{0} w_{2}\right)
$$

which gives

$$
\mathcal{H} B\left(w_{1}, w_{2}\right)-B\left(w_{1}, \mathcal{H} w_{2}\right)=\pi_{0}\left(w_{1} w_{2}\right)+w_{1} \pi_{0} w_{2}-2\left(\pi_{0} w_{1}\right)\left(\pi_{0} w_{2}\right)
$$

and property 1 is proved.

Proof of property 2: In (3) we have in particular (see [6])

$$
\begin{aligned}
\mathcal{N}_{2}(w, w) & =\partial_{t}\left[\dot{w}, w^{\prime}\right]+\partial_{x}\left\{\mathcal{H}\left(\dot{w}^{2}\right)-3 \dot{w} \mathcal{H} \dot{w}\right\} \\
\mathcal{N}_{3}(w, w, w) & =-\mathcal{H} \partial_{x}\left\{4(\mathcal{H} \dot{w})\left(\mathcal{H}\left(w^{\prime} \mathcal{H} \dot{w}\right)\right\}+w^{\prime \prime}\left(\pi_{0} \dot{w}\right)^{2} .\right.
\end{aligned}
$$

Then we obtain

$$
\begin{aligned}
\mathcal{N}_{2}(w, w) & =\ddot{w} \mathcal{H} w^{\prime}-w^{\prime} \mathcal{H} \ddot{w}-2 \dot{w} \mathcal{H} \dot{w}^{\prime}-4 \dot{w}^{\prime} \mathcal{H} \dot{w}+2 \mathcal{H}\left(\dot{w} \dot{w}^{\prime}\right) \\
& =B\left(\ddot{w}, w^{\prime}\right)+2 B\left(\dot{w}, \dot{w}^{\prime}\right)-B\left(w^{\prime}, \ddot{w}\right)
\end{aligned}
$$

hence property 2 is proved. This ends the proof of lemma.1. 


\section{Generalized formal standing waves}

In this section we show the main result of the paper:

Theorem 5 The solutions of (1) taking the form of formal expansions in powers of the amplitude $\varepsilon$, are such that

$$
w=\sum_{n \geq 1} \varepsilon^{n} w^{(n)}, \quad \mu=\varepsilon^{2} / 4
$$

with any choice of finite subset $I$ of $\mathbb{N}$

$$
w^{(1)}=\sum_{q \in I} \frac{\varepsilon_{q}}{q^{2}} \cos q^{2} x \cos q t, \quad \varepsilon_{q}= \pm 1,
$$

and

$$
\begin{aligned}
w^{(2)}= & -\sum_{\substack{r, s \in I \\
r^{2}}} \frac{\varepsilon_{r} \varepsilon_{s}}{r^{2}} \cos \left(r^{2}+s^{2}\right) x \cos r t \cos s t+\sum_{r \in I} \frac{1}{4 r^{2}} \cos 2 r t+ \\
& +\sum_{\substack{r, s \in I \\
r>s}} \frac{\varepsilon_{r} \varepsilon_{s}}{s^{2}} \cos \left(r^{2}-s^{2}\right) x \cos r t \cos s t
\end{aligned}
$$

Remark: Even though $I$ infinite gives formally a sequence $w^{(n)}$, it appears in such a case that in some parts of the computation unbounded terms would occur. The question of the relevance of such solutions would imply a formulation weaker than the one we are taking here.

Proof of the theorem: Let us consider the equation

$$
\mathcal{E}(v, \mu)=0
$$

and decompose formally

$$
\begin{aligned}
v & =\Theta+\Upsilon, \quad \Theta=P_{0} v, \Upsilon=Q_{0} v \\
\Theta & =\sum_{q \in \mathbb{N}} A_{q} \cos q^{2} x \cos q t, \quad \Upsilon_{q^{2}}^{(q)}=0
\end{aligned}
$$

where $v_{n}^{(q)}$ is the coefficient of $\cos n x \cos q t$ in the Fourier expansion of $v$. Then (17) becomes formally

$$
\begin{aligned}
& Q_{0} \mathcal{E}(\Theta+\Upsilon, \mu)=0 \\
& P_{0} \mathcal{E}(\Theta+\Upsilon, \mu)=0
\end{aligned}
$$

where formally

$$
\partial_{\Upsilon} Q_{0} \mathcal{E}(0,0)=\mathcal{L}_{0}
$$

which is formally invertible in the subspace of smooth functions orthogonal to ker $\mathcal{L}_{0}$ in

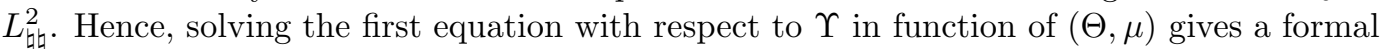
solution

$$
\Upsilon=\mathcal{Y}(\Theta, \mu)=O\left(\|\Theta\|^{3}\right)
$$

which can be put into the second equation instead of $\Upsilon$, for getting the following (formal) bifurcation equation:

$$
\mathcal{G}(\Theta, \mu) \stackrel{\text { def }}{=} P_{0} \mathcal{E}(\Theta+\mathcal{Y}(\Theta, \mu), \mu)=0
$$

where

$$
\mathcal{G}(\Theta, \mu)=-\mu \mathcal{H} \Theta^{\prime}+P_{0}\left\{\mathcal{N}_{3}(\Theta, \Theta, \Theta)+\partial_{x} B\left(\Theta, \mathcal{N}_{2}(\Theta, \Theta)\right)\right\}+O\left(\|\Theta\|^{4}\right) .
$$


In fact, we can show the following fundamental (and simple) result probably related to the integrability of the cubic normal form of the system put into a Hamiltonian formulation (see Craig and Worfolk [3])

\section{Lemma 6 For}

$$
\Theta=\sum_{q \in \mathbb{N}} A_{q} \cos q^{2} x \cos q t
$$

the cubic order terms of the bifurcation equation satisfy formally

$$
P_{0}\left\{\mathcal{N}_{3}(\Theta, \Theta, \Theta)+\partial_{x} B\left(\Theta, \mathcal{N}_{2}(\Theta, \Theta)\right)\right\}=-\frac{1}{4} \sum_{q \in \mathbb{N}} q^{6} A_{q}^{3} \cos q^{2} x \cos q t
$$

Proof: see Appendix 1.

End of the proof of the theorem: Now setting $\mu=\varepsilon^{2} / 4$, we see from the form of (19) that

$$
\mathcal{G}\left(\varepsilon \Theta, \frac{\varepsilon^{2}}{4}\right)=\varepsilon^{3}\left\{\mathcal{G}_{0}(\Theta)+\varepsilon \widetilde{\mathcal{G}}(\Theta, \varepsilon)\right\}
$$

and from Lemma 6

$$
\mathcal{G}_{0}(\Theta)=\frac{1}{4} \sum_{q \in \mathbb{N}}\left(1-q^{4} A_{q}^{2}\right) q^{2} A_{q} \cos q^{2} x \cos q t .
$$

Let us take any finite subset $I$ of $\mathbb{N}$, and define

$$
\Theta^{(1)}=\sum_{q \in I} \frac{\varepsilon_{q}}{q^{2}} \cos q^{2} x \cos q t
$$

where $\varepsilon_{q}= \pm 1$, then $\mathcal{G}_{0}\left(\Theta^{(1)}\right)=0$. We can then look for a formal expansion in powers of $\varepsilon$, solution of

$$
\mathcal{G}\left(\Theta, \varepsilon^{2} / 4\right)=0
$$

under the form

$$
\Theta=\sum_{p \geq 1} \varepsilon^{p} \Theta^{(p)} .
$$

All $\Theta^{(p)}, p \geq 2$ may be computed iteratively, since at each step we just need to invert the operator $\partial_{\Theta} \mathcal{G}_{0}\left(\Theta^{(1)}\right)$ defined for

$$
\theta=\sum_{q \in \mathbb{N}} a_{q} \cos q^{2} x \cos q t
$$

by

$$
\begin{aligned}
\left\{\partial_{\Theta} \mathcal{G}_{0}\left(\Theta^{(1)}\right) \theta\right\}_{q} & =\frac{1}{4}\left(q^{2}-3 q^{6} A_{q}^{2}\right) a_{q} \\
& =\left\{\begin{array}{c}
-\frac{q^{2}}{2} a_{q} \text { if } q \in I \\
\frac{q^{2}}{4} a_{q} \text { if } q \notin I
\end{array} .\right.
\end{aligned}
$$

This linear operator is indeed formally invertible. Coming back to the corresponding formal solutions $w$ of (1), we can therefore compute the expansion (14) with any form (15) of $w^{(1)}$. The computation of $w^{(2)}$ is useful for the proof of existence of these solutions. A simple identification at order $\varepsilon^{2}$ gives

$$
\mathcal{L}_{0} w^{(2)}+\mathcal{N}_{2}\left(w^{(1)}, w^{(1)}\right)=0,
$$


which is already computed (see (20))

$$
\begin{aligned}
\mathcal{N}_{2}\left(w^{(1)}, w^{(1)}\right)= & \sum_{r, s \in I} \varepsilon_{r} \varepsilon_{s} \frac{s}{r} \cos \left(r^{2}+s^{2}\right) x\{\cos (r-s) t-\cos (r+s) t\}+ \\
& +\sum_{r, s \in I, r>s} \varepsilon_{r} \varepsilon_{s} \frac{1}{s} \cos \left(r^{2}-s^{2}\right) x \frac{d}{d t}\{\sin (r+s) t-\sin (r-s) t\}+ \\
& +\sum_{r \in I} \cos 2 r t,
\end{aligned}
$$

where we observe that the finiteness of the set $I$ allows to define $\mathcal{N}_{2}\left(w^{(1)}, w^{(1)}\right)$. The result of the theorem is then straightforward.

\section{Appendix 1}

In this appendix, we prove lemma 6 . First we compute $\mathcal{N}_{2}(\Theta, \Theta)$ :

$$
\begin{aligned}
\mathcal{N}_{2}(\Theta, \Theta)= & \sum_{r, s} A_{r} A_{s} r s^{3} \cos \left(r^{2}+s^{2}\right) x\{\cos (r-s) t-\cos (r+s) t\}+ \\
& +\sum_{r>s} A_{r} A_{s} r^{2} s \cos \left(r^{2}-s^{2}\right) x \frac{d}{d t}\{\sin (r+s) t-\sin (r-s) t\}+ \\
& +\sum_{r} A_{r}^{2} r^{4} \cos 2 r t .
\end{aligned}
$$

Now, for $P_{0} \partial_{x} B\left(\Theta, \mathcal{N}_{2}(\Theta, \Theta)\right)$ we use the following property 3 of $B$

$$
\left\langle B\left(w_{1}, w_{2}\right), w_{3}\right\rangle=\left\langle B\left(w_{1}, w_{3}\right), w_{2}\right\rangle,
$$

from which, we obtain

$$
\begin{aligned}
P_{0} \partial_{x} B\left(\Theta, \mathcal{N}_{2}(\Theta, \Theta)\right) & =\frac{1}{\pi^{2}}\left\langle\left\langle\partial_{x} B\left(\Theta, \mathcal{N}_{2}(\Theta, \Theta)\right), \cos q^{2} x \cos q t\right\rangle\right\rangle \\
& =\frac{q^{2}}{\pi^{2}}\left\langle\left\langle B\left(\Theta, \sin q^{2} x \cos q t\right), \mathcal{N}_{2}(\Theta, \Theta)\right\rangle\right\rangle .
\end{aligned}
$$

We then need to consider $B\left(\Theta, \sin q^{2} x \cos q t\right)$ :

$$
\begin{aligned}
B\left(\Theta, \sin q^{2} x \cos q t\right)= & \sum_{p} A_{p} \cos \left(p^{2}+q^{2}\right) x \cos p t \cos q t+\frac{1}{2} A_{q} \cos ^{2} q t+ \\
& +\sum_{p<q} A_{p} \cos \left(q^{2}-p^{2}\right) x \cos p t \cos q t .
\end{aligned}
$$

Let us now consider $P_{0} \mathcal{N}_{3}(\Theta, \Theta, \Theta)$. We have from the definition (13) of $\mathcal{N}_{3}$

$$
\begin{aligned}
\left\{P_{0} \mathcal{N}_{3}(\Theta, \Theta, \Theta)\right\}_{q} & =\frac{1}{\pi^{2}}\left\langle\left\langle\mathcal{N}_{3}(\Theta, \Theta, \Theta), \cos q^{2} x \cos q t\right\rangle\right\rangle \\
& =\frac{q^{2}}{\pi^{2}}\left\langle\left\langle 2 \mathcal{H}\left(w^{\prime} \mathcal{H} \dot{w}\right), 2(\mathcal{H} \dot{w}) \cos q^{2} x \cos q t\right\rangle\right\rangle
\end{aligned}
$$

since $\pi_{0} \Theta=0$, so we obtain

$$
\begin{aligned}
2 \mathcal{H}\left(w^{\prime} \mathcal{H} \dot{w}\right)= & -\sum_{r, s} r s^{2} A_{r} A_{s} \sin \left(r^{2}+s^{2}\right) x \sin r t \cos s t+ \\
& +\sum_{r>s} r s A_{r} A_{s} \sin \left(r^{2}-s^{2}\right) x \frac{d}{d t}(\sin r t \sin s t)
\end{aligned}
$$


and

$$
2(\mathcal{H} \dot{w}) \cos q^{2} x \cos q t=\sum_{p} p A_{p}\left\{\sin \left(p^{2}+q^{2}\right) x+\sin \left(p^{2}-q^{2}\right) x\right\} \sin p t \cos q t .
$$

Now we finish the calculations of

$$
\frac{1}{\pi^{2}}\left\langle\left\langle B\left(\Theta, \sin q^{2} x \cos q t\right), \mathcal{N}_{2}(\Theta, \Theta)\right\rangle\right\rangle
$$

and

$$
\frac{1}{\pi^{2}}\left\langle\left\langle 2 \mathcal{H}\left(w^{\prime} \mathcal{H} \dot{w}\right), 2(\mathcal{H} \dot{w}) \cos q^{2} x \cos q t\right\rangle\right\rangle,
$$

noting that for computing the integrals and ending the proof of lemma 5 , we use the following lemma (elementary algebra):

Lemma 7 (nonresonant coefficients) The set of positive integers which satisfy

$$
\begin{aligned}
\text { (i) } q^{2}+p^{2} & =r^{2}+s^{2} \quad \text { or } \\
\text { (ii) } q^{2}+p^{2} & =r^{2}-s^{2} \\
\text { with } q \pm p \pm r \pm s & =0,
\end{aligned}
$$

then also satisfies (resp.)

$$
\begin{aligned}
\text { (i) } q & =r, \quad p=s, \text { or } q=s, \quad p=r \\
\text { (ii) } q+p & =r+s, \text { or } q+r=p+s, \text { or } q+s=p+r .
\end{aligned}
$$

Using the above expressions in the calculation of the scalar products for

$$
\frac{1}{\pi^{2}}\left\langle\left\langle B\left(\Theta, \sin q^{2} x \cos q t\right), \mathcal{N}_{2}(\Theta, \Theta)\right\rangle\right\rangle+\frac{1}{\pi^{2}}\left\langle\left\langle 2 \mathcal{H}\left(w^{\prime} \mathcal{H} \dot{w}\right), 2(\mathcal{H} \dot{w}) \cos q^{2} x \cos q t\right\rangle\right\rangle
$$

we can show that the sum of the contribution of the simple terms and of all terms where part (i) of the above lemma applies is

$$
-\frac{1}{4} q^{4} A_{q}^{3}
$$

It then remains to compute the terms where part (ii) of the above lemma applies, and it can be shown (after symmetrization) that these terms cancel. This ends the proof of lemma 6 .

\section{$5 \quad$ Appendix 2}

In this Appendix we prove theorem 2. Let us consider the mapping $F: w \rightarrow v$ defined by (6), it is easily seen that $F: H_{\natural}^{s} \mapsto H_{\natural}^{s-1}$ is an analytic mapping for any $s>2$. We first need three auxiliary lemmas.

Lemma 8 Let $1<\alpha$ and

$$
M(\xi)=\sum_{\eta \in \mathbb{Z}^{2}} f(\xi-\eta) g(\eta), \quad f, g \geq 0,
$$

then

$$
\sum_{\xi \in \mathbb{Z}^{2}}\left(1+\xi^{2}\right)^{\alpha} M(\xi)^{2} \leq c(\alpha)\left(\sum_{\xi \in \mathbb{Z}^{2}}\left(1+\xi^{2}\right)^{\alpha} f(\xi)^{2}\right)\left(\sum_{\xi \in \mathbb{Z}^{2}}\left(1+\xi^{2}\right)^{\alpha} g(\xi)^{2}\right) .
$$


Proof. This is just a rephrasing of the classical fact that the space $H_{\text {㛣 }}^{\alpha}$ is an algebra when $\alpha>1$.

Lemma 9 Let $s>2$ and $s \geq 0$, and let us define

$$
[\mathcal{H}, a] u=\mathcal{H}(a u)-a \mathcal{H} u
$$

then the inequality

$$
\left\|[\mathcal{H}, a] u^{\prime}\right\|_{H^{s}} \leq c(r)\|a\|_{H^{s}}\|u\|_{H^{s}}
$$

holds for any $a \in H_{\text {吼 }}^{s}$ and $u \in H_{\text {蚂. }}^{s}$.

Proof. It is easy to see that (with $\eta=\left(\eta_{1}, \eta_{2}\right)$ )

$$
\left[\widehat{\mathcal{H}, a]} u^{\prime}(\xi)=-\sum_{\eta \in \mathbb{Z}^{2}}\left(\operatorname{sgn} \xi_{1}-\operatorname{sgn} \eta_{1}\right) \eta_{1} \hat{a}(\xi-\eta) \hat{u}(\eta)\right.
$$

We then use the inequalities

$$
\begin{aligned}
\left(1+\left|\eta_{1}\right|\right)\left|\operatorname{sgn} \xi_{1}-\operatorname{sgn} \eta_{1}\right| & \leq 2\left(1+\left|\xi_{1}-\eta_{1}\right|\right), \\
(1+|\xi|)^{s} & \leq c(1+|\xi-\eta|)^{s}+c(1+|\eta|)^{s}
\end{aligned}
$$

which leads to

$$
\begin{aligned}
\sum_{\xi \in \mathbb{Z}^{2}}\left(1+\xi^{2}\right)^{s}\left|\widehat{\mathcal{H}, a]} u^{\prime}\right|^{2} \leq & 4 c^{2} \sum_{\eta \in \mathbb{Z}^{2}}\left(1+|\xi-\eta|^{2}\right)^{s}|\hat{a}(\xi-\eta)|^{2}\left(\sum_{\eta \in \mathbb{Z}^{2}}|\eta||\hat{u}(\eta)|\right)^{2}+ \\
& +4 c^{2}\left(\sum_{\eta \in \mathbb{Z}^{2}}(1+|\xi-\eta|)|\hat{a}(\xi-\eta)|\right)^{2} \sum_{\eta \in \mathbb{Z}^{2}}\left(1+|\eta|^{2}\right)^{s}|\hat{u}(\eta)|^{2}
\end{aligned}
$$

It remains to note that for $s>2$,

$$
\sum_{\eta \in \mathbb{Z}^{2}}\left|\eta\|\hat{u}(\eta) \mid \leq c(s)\| u \|_{H^{s}}\right.
$$

and the lemma follows.

Lemma 10 For any $2<s \leq p$ there is a positive constant $C(s, p)$ depending on $s, p$ only, such that if $a \in H_{\text {吼 }}^{p}$ and $C(s, p)\|a\|_{H^{p}}<1$, then the equation

$$
a(x) u^{\prime}(x)+u(x)=v(x), \quad v \in H_{\natural}^{s}
$$

has a unique solution $u \in H_{\text {如 }}^{s}$ satisfying the inequality

$$
\|u\|_{H^{s}} \leq\left(1-C(s, p)\|a\|_{H^{p}}\right)^{-1}\|v\|_{H^{s}} .
$$

Proof. Without loss of generality we can assume that $a, v \in C^{\infty}\left(\mathbb{R}^{2}\right)$. Consider the second order equation

$$
-\varepsilon u^{\prime \prime}+a u^{\prime}+u=v
$$

with a small positive parameter $\varepsilon$. Multiplying both sides by $u$ and integrating over a period we obtain

$$
\int_{-\pi}^{\pi} \int_{-\pi}^{\pi}\left(2 \varepsilon u^{\prime 2}+\left(2-a^{\prime}\right) u^{2}\right) d x d t=2 \int_{-\pi}^{\pi} \int_{-\pi}^{\pi} v u d x d t .
$$


For $a$ satisfying the inequality $\left|a^{\prime}\right|<2$ we have $\|u\|_{0} \leq c\|v\|_{0}$. It follows from this and general theory of second-order ODE that for a such $a$, equation (24) has a unique solution $u_{\varepsilon} \in C^{\infty}$. Now we need a priori estimates for $u_{\varepsilon}$.

Multiplying both sides of (24) by $(1-\Delta)^{s} u, \Delta=\partial_{x}^{2}+\partial_{t}^{2}$, and integrating by parts we obtain

$$
\int_{-\pi}^{\pi} \int_{-\pi}^{\pi}\left(2 \varepsilon w^{\prime 2}+\left(2-a^{\prime}\right) w^{2}+R w\right) d x=2 \int_{-\pi}^{\pi} \int_{-\pi}^{\pi}(1-\Delta)^{s / 2} v w d x
$$

where

$$
R=2(1-\Delta)^{s / 2}\left(a u^{\prime}\right)-2 a(1-\Delta)^{s / 2} u^{\prime}, \quad w=(1-\Delta)^{s / 2} u
$$

Note that

$$
\hat{R}(\xi)=2 i \sum_{\eta \in \mathbb{Z}^{2}}\left[\left(1+\xi^{2}\right)^{s / 2}-\left(1+\eta^{2}\right)^{s / 2}\right] \eta_{1} \hat{a}(\xi-\eta) \hat{u}(\eta),
$$

which gives

$$
|\hat{R}(\xi)| \leq c \sum_{\eta \in \mathbb{Z}^{2}}\left[\left(1+\xi^{2}\right)^{(s-1) / 2}+\left(1+\eta^{2}\right)^{(s-1) / 2}\right]|\eta-\xi \| \hat{a}(\xi-\eta)||\eta \hat{u}(\eta)| \leq I_{1}(\xi)+I_{2}(\xi)
$$

where

$$
\begin{aligned}
& I_{1}(\xi)=c\left(1+\xi^{2}\right)^{(s-1) / 2} \sum_{\eta \in \mathbb{Z}^{2}}|\eta-\xi \|| \hat{a}(\xi-\eta)|| \eta \hat{u}(\eta) \mid \\
& I_{2}(\xi)=c \sum_{\eta \in \mathbb{Z}^{2}}|\eta-\xi||\hat{a}(\xi-\eta)|\left(1+\eta^{2}\right)^{s / 2}|\hat{u}(\eta)| .
\end{aligned}
$$

From Lemma 8 with $\alpha=s-1$, and since $s \leq p$, we conclude that

$$
\left(\sum_{\xi \in \mathbb{Z}^{2}} I_{1}(\xi)^{2}\right)^{1 / 2} \leq c(s, p)\|u\|_{H^{s}}\|a\|_{H^{p}}
$$

On the other hand, the standard convolution estimate yields

$$
\left(\sum_{\xi \in \mathbb{Z}^{2}} I_{2}(\xi)^{2}\right)^{1 / 2} \leq c \sum_{\xi \in \mathbb{Z}^{2}}|\xi||\hat{a}(\xi)|\left(\sum_{\xi \in \mathbb{Z}^{2}}\left(1+\xi^{2}\right)^{s}|\hat{u}(\xi)|^{2}\right)^{1 / 2}
$$

Noting the estimate (22) for $p>2$, we conclude from (27) that

$$
\left(\sum_{\xi \in \mathbb{Z}^{2}} I_{2}(\xi)^{2}\right)^{1 / 2} \leq c(s, p)\|u\|_{H^{s}}\|a\|_{H^{p}}
$$

Combining (26) and (28) we obtain

$$
\|R\|_{L^{2}}=\left(\sum_{\xi \in \mathbb{Z}^{2}}|\widehat{R}(\xi)|^{2}\right)^{1 / 2} \leq c(s, p)\|u\|_{H^{s}}\|a\|_{H^{p}} .
$$

From (29), (25) and inequalities

$$
\left|a^{\prime}\right| \leq c(p)\|a\|_{H^{p}}, \quad\|w\|_{L^{2}}=\|u\|_{H^{s}}
$$


we obtain

$$
2 \varepsilon\left\|u^{\prime}\right\|_{H^{s}}^{2}+\left(2-c(p)\|a\|_{H^{p}}\right)\|w\|_{L^{2}}^{2}-\|R\|_{L^{2}}\|w\|_{L^{2}} \leq 2\|v\|_{H^{s}}\|w\|_{L^{2}}
$$

or

$$
\varepsilon\left\|u^{\prime}\right\|_{H^{s}}^{2}+\left(1-C(s, p)\|a\|_{H^{p}}\right)\|u\|_{H^{s}}^{2} \leq\|v\|_{H^{s}}\|u\|_{H^{s}} .
$$

Thus, for $1-C(s, p)\|a\|_{H^{p}}>0$, we get the estimate for $u_{\varepsilon}$

$$
\left(1-C(s, p)\|a\|_{H^{p}}\right)\left\|u_{\varepsilon}\right\|_{H^{s}} \leq\|v\|_{H^{s}} .
$$

Hence for $1-C(s, p)\|a\|_{H^{p}}>0$ solutions of the regularized problem (24) are bounded in $H_{\text {如 }}^{s}$ and we can suppose that they converge weakly in this space to some $u \in H_{\text {如. Passing }}^{s}$ to the limit in the integral identity

$$
\int_{-\pi}^{\pi} \int_{-\pi}^{\pi}\left(\varepsilon u_{\varepsilon}^{\prime} \varphi^{\prime}-u_{\varepsilon}(a \varphi)^{\prime}+u_{\varepsilon} \varphi-v \varphi\right) d x=0
$$

we conclude that $u$ satisfies equation (23) and the lemma follows.

Proof of Theorem 2. We use a variant of the Newton iteration method for finding the inverse of the mapping $F$. Fix $v \in H_{\text {吅 }}^{p}, p>3$ and consider the sequence of functions $w_{n}$ defined by $w_{0}=0$,

$$
-2\left(\mathcal{H} w_{n-1}\right) w_{n}^{\prime}+w_{n}=v-\left[\mathcal{H}, w_{n-1}\right] w_{n-1}^{\prime}, \quad n \geq 1
$$

Note that, by Lemma 9,

$$
\left\|\left[\mathcal{H}, w_{n-1}\right] w_{n-1}^{\prime}\right\|_{H^{p}} \leq c_{1}\left\|w_{n-1}\right\|_{H^{p}}^{2},
$$

Set $c_{2}=2 C(p, p)$, where $C(p, p)$ is a constant from Lemma 10. If $c_{2}\left\|w_{n-1}\right\|_{H^{p}}<1$ we have, by Lemma 10 ,

$$
\left\|w_{n}\right\|_{H^{p}} \leq\left(1-c_{2}\left\|w_{n-1}\right\|_{H^{p}}\right)^{-1}\left(\|v\|_{H^{p}}+c_{1}\left\|w_{n-1}\right\|_{H^{p}}^{2}\right) .
$$

It follows from this that for any $v$ satisfying the inequality

$$
\|v\|_{H^{p}} \leq r=\frac{1}{8\left(c_{1}+c_{2}\right)}
$$

the functions $w_{n}$ have the bound

$$
\left\|w_{n}\right\|_{H^{p}} \leq \frac{1-\sqrt{1-4\left(c_{1}+c_{2}\right)\|v\|_{H^{p}}}}{2\left(c_{1}+c_{2}\right)} \leq \frac{2}{1+\sqrt{\frac{1}{2}}}\|v\|_{H^{p}}<1 /\left(2 c_{2}\right) .
$$

Hence for all $v \in B_{p}(r)$ the sequence $w_{n}$ is well defined and satisfies inequality (??). Next note that

$$
\begin{gathered}
-\left(2 \mathcal{H} w_{n-1}\right)\left(w_{n}-w_{n-1}\right)^{\prime}+\left(w_{n}-w_{n-1}\right)= \\
=-\left(2 \mathcal{H}\left(w_{n-2}-w_{n-1}\right)\right) w_{n-1}^{\prime}-\left[\mathcal{H}, w_{n-1}-w_{n-2}\right] w_{n-1}^{\prime}-\left[\mathcal{H}, w_{n-2}\right]\left(w_{n-1}^{\prime}-w_{n-2}^{\prime}\right) .
\end{gathered}
$$

Applying Lemmas 10 and 9, we obtain

$$
\left\|w_{n}-w_{n-1}\right\|_{H^{p-1}} \leq 2 c_{3}\left(\left\|w_{n-1}\right\|_{H^{p}}+\left\|w_{n-2}\right\|_{H^{p}}\right)\left\|w_{n-1}-w_{n-2}\right\|_{H^{p-1}}
$$

where $c_{3}$ depends on p only. From this and (33) we conclude that for $v \in B_{p}(r)$,

$$
\left\|w_{n}-w_{n-1}\right\|_{H^{p-1}} \leq r \frac{8 c_{3}}{1+\sqrt{\frac{1}{2}}}\left\|w_{n-1}-w_{n-2}\right\|_{H^{p-1}}
$$


It follows from this that there is $r>0$, depending on $p$ only such that for $v \in B_{p}(r)$,

$$
\left\|w_{n}-w_{n-1}\right\|_{H^{p-1}} \leq \frac{1}{2}\left\|w_{n-1}-w_{n-2}\right\|_{H^{p-1}} .
$$

It follows from this and (33) that $w_{n} \rightarrow w \in B_{p}(2 r)$ as $n \rightarrow \infty$. Hence there exists an inverse mapping

$$
w=F^{-1}(v) \equiv G(v), \quad G: B_{p}(r) \mapsto B_{p}(2 r),
$$

and from (33), we have

$$
\|w\|_{H^{p}} \leq 2\|v\|_{H^{p}}
$$

Next, let us show that for $w \in H_{\text {如 }}^{p}$, the derivative $\partial_{w} F(w)$ which is in $\mathcal{L}\left(H_{\text {如 }}^{p}, H_{\text {吅 }}^{p-1}\right)$ has an inverse in $\mathcal{L}\left(H_{\text {煰 }}\right)$ for $2<s \leq p-1$, and $\|w\|_{H^{p}}$ small enough. Indeed, we can write for $w \in H_{\text {如 }}^{p}$ and any $W \in H_{\text {如 }}^{p}$

$$
\begin{aligned}
\partial_{w} F(w) & =A_{1}(w)+A_{2}(w), \\
A_{1}(w) W & =-2(\mathcal{H} w) W^{\prime}+W \\
A_{2}(w) W & =[\mathcal{H}, w] W^{\prime}+B\left(W, w^{\prime}\right) .
\end{aligned}
$$

By Lemma 9 and (33), we have

$$
\left\|A_{2}(w) W\right\|_{H^{s-1}} \leq c_{4} r\|W\|_{H^{s-1}}, \quad 3<s \leq p .
$$

On the other hand, Lemma 10 along with (33) implies the existence of the inverse $A_{1}^{-1}$ : $H_{\text {吅 }}^{s-1} \mapsto H_{\text {吅 }}^{s-1}$ satisfying the inequality

$$
\left\|A_{1}^{-1} u\right\|_{H^{s-1}} \leq 2\|u\|_{H^{s-1}}, \quad 3<s \leq p .
$$

Using the identity $\partial_{w} F(w)^{-1}=\left(1+A_{1}^{-1} A_{2}\right)^{-1} A_{1}^{-1}$ we then obtain, for $r<1 / 4 c_{4}$,

$$
\left\|\partial_{w} F(w)^{-1}\right\|_{\mathcal{L}\left(H_{\text {吼 }}^{s-1}\right)} \leq 4 .
$$

Let us now fix $r<r(n+p), v \in B_{p+n}(r)$ and $h \in H_{\text {吼 }}^{p+n}$ such that $v+h \in B_{p+n}(r)$ and show that $k$-linear operators $G_{k}(v)$ are well defined and satisfy (9). We already proved that this is satisfied for $k=1$. Suppose that the assertion is fulfilled for $G_{1}, \ldots, G_{k-1}$ satisfying inequalities (9) and prove that it holds true for $G_{k}$. Since $p>3$, we have for $2<s \leq p+n-k$

$$
\begin{gathered}
\left\|\Re_{k}\right\|_{H^{s}} \leq c(p, n) \sum_{i+j=k ; i, j \leq k-1}\left\|G_{i}(v)(h)\right\|_{H^{s}}\left\|G_{j}(v)(h)\right\|_{H^{s+1}} \leq \\
c(p, n) \sum_{i+j=k ; i, j \leq k-1}\|h\|_{H^{s+i-1}}^{i}\|h\|_{H^{s+j}}^{j} \leq c(p, n)\|h\|_{H^{s+k-1}}^{k} .
\end{gathered}
$$

From this, inequality (39), and relations (10) we conclude that $G_{k}$ satisfies (9) as said in theorem 2. Our next task is to estimate the rest in (8). Set

$$
w_{h}=G(v+h), \quad w=G(v), \quad O_{n}=w_{h}-w-\sum_{k=1}^{n} G_{k}(v)(h) .
$$

It follows from (9) that

$$
\left\|O_{n}\right\|_{H^{p}} \leq\left\|w_{h}\right\|_{H^{p}}+\|w\|_{H^{p}}+\sum_{k=1}^{n}\left\|G_{k}(v)(h)\right\|_{H^{p}} \leq 4 r(p)+c\|h\|_{H^{n+p-1}} \leq \operatorname{cr}(p) .
$$


Substituting (40) into equality $F\left(w_{h}\right)-F(w)=h$ yields

$$
\partial_{w} F\left(w_{h}\right) O_{n}-B\left(O_{n}, O_{n}^{\prime}\right)=-\sum_{i+j>n ; i, j \leq n-1} B\left(G_{i}, G_{j}^{\prime}\right),
$$

where $w=G(v)$. Note that

$\sum_{i+j>n ; i, j \leq n-1}\left\|B\left(G_{i}, G_{j}^{\prime}\right)\right\|_{H^{p-1}} \leq \sum_{i+j>n ; i, j \leq n-1}\left\|G_{i}(v)(h)\right\|_{H^{p-1}}\left\|G_{j}(v)(h)\right\|_{H^{p}} \leq c\|h\|_{H^{p+n-1}}^{n+1}$,

and

$$
\left\|B\left(O_{n}, O_{n}^{\prime}\right)\right\|_{H^{p-1}} \leq c\left\|O_{n}\right\|_{H^{p-1}}\left\|O_{n}\right\|_{H^{p}} \leq \operatorname{cr}(p)\left\|O_{n}\right\|_{H^{p-1}}
$$

Combining (39), (43),(44) we obtain

$$
\left\|O_{n}\right\|_{H^{p-1}} \leq c(1-4 c r(p))^{-1}\|h\|_{H^{p+n-1}}^{n+1} \leq c\|h\|_{H^{p+n-1}}^{n+1} .
$$

and the theorem follows.

For showing the first estimate of Corollary 3, we use again lemmas 9 and 10 with $w \in$ $H_{\text {吼 }}^{s+1}, f \in H_{\text {叫 }}^{s}, s>2$ in the differential equation

$$
-2(\mathcal{H} w) f^{\prime}+f=e-\partial_{x}([\mathcal{H}, w] f)+2 f \mathcal{H} w^{\prime} .
$$

The last estimate in the Corollary 3 is then straightforward.

\section{References}

[1] C.J.Amick, J.F.Toland. The semi-analytic theory of standing waves. Proc. Roy. Soc. Lond. A 411 (1987), 123-138.

[2] P.J.Bryant, M.Stiassnie. Different forms for nonlinear standing waves in deep water. J.Fluid Mech. 272 (1994), 135-156.

[3] W.Craig, P.Worfolk. An integrable normal form for water waves in infinite depth. Physica D 84 (1995), 513-531.

[4] A.I.Dyachenko, E.A.Kuznetsov, M.D.Spector, V.E.Zakharov. Analytic description of the free surface dynamics of an ideal fluid. Physics Lett. A 221 (1996), 73-79.

[5] G.Iooss. On the standing wave problem in deep water. J.Math. Fluid Mech. 4 (2002), $155-185$.

[6] G.Iooss, P.I.Plotnikov, J.F.Toland. Standing waves on an infinitely deep perfect fluid under gravity. preprint 2004.

[7] G.W.Penney, A.T.Price. Finite periodic stationary gravity waves in a perfect fluid. Phil. trans. roy. Soc. A, 244 (1952), 254-284.

[8] L.W.Schwartz, A.K.Whitney. A semi-analytic solution for nonlinear standing waves in deep water. J. Fluid Mech. 107 (1981), 147-171. 